\title{
Corela
}

Cognition, représentation, langage

HS-8 | 2010

L'interpellation

\section{Quand et comment survient l'interpellation en classe : étude comparative d'oraux en maternelle et au collège}

Emmanuèle Auriac et Mylène Blasco-Dulbecco

\section{(2) OpenEdition \\ Journals}

Édition électronique

URL : http://journals.openedition.org/corela/1692

DOI : 10.4000/corela.1692

ISSN : 1638-573X

Éditeur

Cercle linguistique du Centre et de l'Ouest - CerLICO

Référence électronique

Emmanuèle Auriac et Mylène Blasco-Dulbecco, « Quand et comment survient l'interpellation en classe : étude comparative d'oraux en maternelle et au collège », Corela [En ligne], HS-8 | 2010, mis en ligne le 02 décembre 2010, consulté le 19 avril 2019. URL : http://journals.openedition.org/ corela/1692 ; DOI : 10.4000/corela.1692

Ce document a été généré automatiquement le 19 avril 2019

\section{(c) (i) (3)}

Corela - cognition, représentation, langage est mis à disposition selon les termes de la licence Creative Commons Attribution - Pas d'Utilisation Commerciale - Partage dans les Mêmes Conditions 4.0 International. 


\title{
Quand et comment survient l'interpellation en classe : étude comparative d'oraux en maternelle et au collège
}

\author{
Emmanuèle Auriac et Mylène Blasco-Dulbecco
}

\section{Introduction}

1 Notre recherche intéresse la didactique. Nous proposons d'étudier, de manière comparative, les espaces interlocutoires correspondant à des « interpellations directes " en classe en ce qu'ils constituent a priori des adressages classiques spécifiques des interactions scolaires (Auriac-Slusarczyk, 2009, Auriac-Slusarczyk \& Morge, soumis). Nous avons étudié l'interpellation dans une perspective double, pragmatique et morphosyntaxique, en pariant sur le fait que ces deux orientations sont complémentaires et convergentes.

\section{L'interpellation: définition prudente}

2 Interpellés, par les experts, sur notre manière de définir la notion d'adressage, nous avons prudemment consulté le dictionnaire afin de délimiter l'usage que nous proposons des termes respectif d'adressage et d'interpellation. Nous situerons ces termes dans l'usage qu'on peut en faire en contexte scolaire. Le champ synonymique associé au vocable « interpellation » est très large et nous reviendrons en conclusion sur cet aspect.

Selon le Petit Robert de la langue française (2007), l'interpellation désigne l'acte « d'adresser brusquement la parole à quelqu'un pour l'interroger ", définition qui reprend l'usage qu'en proposait Benveniste (1966) qui indique qu'interpeller correspond à «l'acte de langage où un interlocuteur s'adresse à un autre pour le provoquer, le convoquer ». Dit autrement, interpeller constitue une forme «d'adressage vif ». Cette définition en terme 
« d'acte de langage » assoit théoriquement la perspective d'étude pragmatique que nous proposons. Nous retenons ensuite deux contextes d'usage pour «interpellation». Tout d'abord, en contexte ordinaire, « l'interpellation est une action : celle d'interpeller, et les synonymes sont apostrophe, interrogation, intervention, question" (Petit Robert, 2007). Ainsi, plusieurs actes de langage peuvent correspondre à une interpellation Pour le deuxième contexte cité dans le Petit Robert, "lors d'une séance publique c'est aussi une demande d'explication formulée par un parlementaire et adressée à un ministre » Là, on s'aperçoit que l'on interpelle l'autre lorsqu'il y a «incompréhension" puisque l'interpellation oblige l'autre à s'expliquer. Il est clair que l'interpellation fait appel à une problématique qui peut s'élargir à souhait. Le choix d'une étude comparatiste, dans une double perspective pragmatique et morphosyntaxique, permet une réduction prudente du champ d'approche. Vu le statut déséquilibré au plan des savoirs entre un enseignant et ses élèves, nul doute que le double éclairage interrogation-question et incompréhension doit trouver matière à s'illustrer dans les situations de classes.

En prenant toutes les précautions définitoires, on pourrait dire qu'il y a interpellation en classe dans la mesure où l'enseignant a une visée d'enseignement/apprentissage: un cours c'est un parcours (discours orienté), et sur ce parcours les individus vont se faire élèves (et l'enseignant deviendra aussi au fil des cours davantage enseignant). Dans un cours on interpelle car justement le cours est un exercice commun finalisé qui doit aboutir, qui doit avancer. On y chemine vers un but. S'y loge alors sans doute une forme d'urgence qui appelle le « vif». On fait plus que s'adresser à... en classe on interpelle. D'un côté interpeller c'est s'adresser "vivement " (la police interpelle) : on est en ce cas du côté de l'apostrophe. Autrui est alors « appelé », voire invectivé : on le met sur le devant de la scène, on le nomme, on le place comme celui qui est choisi pour se manifester. L'élève est sommé de réagir. Il est désigné. D'un autre côté interpeller c'est « adresser une demande d'explication » (le parlementaire interpelle le ministre) : on est là du côté de la question. L'interlocuteur ciblé par la question est alors sommé de se situer face à la question. On lui demande de réagir sur le fond, au-delà d'être ciblé/désigné. L'interpellation mêle ainsi un adressage, qui met en visibilité (Toi/Moi), à une activité intellectuelle, qui place l'interlocuteur dans un espace de résolution obligation: explique ! L'élève est ainsi interrogé.

\section{Spécificité de notre corpus}

5 Notre corpus est composé de deux niveaux: 4 enseignantes en classe de maternelle (français : LO, belge : GB et québécois : SW et $\mathrm{GZ}^{1}$ ) ont chacune d'elles été enregistrées 2 fois l'an lorsqu'elles pratiquaient des oraux réflexifs, sur la base du même support (une historiette à visée philosophique, Daniel, 1997). Pour le collège, 5 enseignants, débutants dans la carrière sont enregistrés lors d'une leçon identique au sens où elle fut préparée à l'IUFM (PLC2 de Sciences physique durant l'année de formation 2001²) : ces enseignants (A,B,C,D \& E) suivent a priori le même scénario mis au point pour la quatrième collège. Ces enseignants débutants comme les enseignantes de maternelle ont été préalablement formés à « interagir » avec leurs élèves. Nous disposons donc d'un corpus permettant une comparaison, mais qui n'a pas été constitué pour les besoins de cette étude. Il nous a toutefois semblé intéressant d'exploiter ce corpus large qui permet d'étudier l'interpellation d'une manière exploratoire tout en bénéficiant d'un angle de comparaison et d'une relative unité dans chacun des niveaux (même supports pour les enseignants qui 
suppose des scénarii de cours moins divergents que sur des supports qui auraient été aléatoires). Ce double corpus permet de détecter quels sont les marqueurs les plus saillants qui pourraient entrer dans la catégorie adressage (classique) vs interpellation (adressage vif).

\section{1. Etude préliminaire automatisée}

6 Au regard de l'ampleur du corpus global, nous avons pratiqué une première étude automatisée de chacune des séances à 'laide du logiciel d'analyse Tropes. (version 6). Ce logiciel a l'avantage de trier automatiquement tous les contextes d'emplois, dont ceux des pronoms. Nous avons eu l'idée de mettre à l'épreuve de la comparaison l'usage de la marque pronominale ' $\mathrm{Tu}$ ' en contexte maternelle vs collège.

7 Nous avons ensuite procédé à un relevé de certains marqueurs jugés « en rapport » avec l'acte de langage d'interpellation. Nous avons été vigilants à :

- la présence des pronoms interpellatifs $(t u)$, de marqueurs interpellatifs évidents (Prénoms) ou à des formes d'auto-interpellation (Moi, Je) ;

- au fait que délimiter l'interpellation supposait de délimiter des « épisodes interpellatifs »;

- au fait que l'interpellation demandait une étude fouillée dans la mesure où les configurations « Tu + Prénom », « Prénom seul », « Prénom + tu », etc. apparaissaient.

\subsubsection{Premier regard sur les corpus : les emplois de tu et de je}

8 L'emploi respectif des pronoms je et tu (relevé systématique et dénombrement de ces marqueurs dans l'ensemble des séances en \% relatif aux autres marqueurs pronominaux : Je, Tu, Il, Nous, Vous, On) dans les différentes séances débouchent sur des premières tendances. En maternelle, quelle que soit la séance (GZ1= séance $n^{\circ} 1 ; \mathrm{GZ2}=$ séance $\left.\mathrm{n}^{\circ} 2\right)$ on observe un équilibre d'usage entre les emplois de je et les emplois de tu. Pour la première séance l'emploi du tu correspond à une moyenne de 19,15\% relativement à la classe des pronoms. Pour la deuxième séance, la moyenne d'emploi des tu est de $20,4 \%$ relativement à la catégorie des pronoms.

Emplois de tu et je en Maternelle

\begin{tabular}{|c|c|c|c|c|}
\hline $\begin{array}{c}\text { Séance } \text { n }^{\circ} 1 \\
\text { GZ 1 }\end{array}$ & LO 1 & SW 1 & GB 1 & Moyenne \\
\hline $\begin{array}{c}\text { Je } \\
23,4 \%\end{array}$ & $13,9 \%$ & $16,3 \%$ & $21,3 \%$ & $18,72 \%$ \\
\hline $\begin{array}{c}\text { Tu } \\
17,4 \%\end{array}$ & $19,6 \%$ & $18 \%$ & $21,6 \%$ & $19,15 \%$ \\
\hline
\end{tabular}

\begin{tabular}{|c|c|c|c|c|}
\hline $\begin{array}{c}\text { Séance n'2 } \\
\mathbf{G Z ~ 2}\end{array}$ & LO 2 & SW 2 & GB 2 & Moyenne \\
\hline $\begin{array}{c}\mathrm{Je} \\
22 \%\end{array}$ & $18,6 \%$ & $15,7 \%$ & $30,8 \%$ & $21,77 \%$ \\
\hline $\begin{array}{c}\mathrm{Tu} \\
22,3 \%\end{array}$ & $21,8 \%$ & $19,1 \%$ & $18,4 \%$ & $20,4 \%$ \\
\hline
\end{tabular}

Par comparaison, au collège l'emploi du tu est moindre corrélativement à l'emploi du je. Sur l'ensemble des séquences on obtient une moyenne d'emploi du pronom interpellatif tu de $14,5 \%$. 


\begin{tabular}{|l|c|c|c|c|c|c|}
\hline Séance unique & $\boldsymbol{A}$ & $\boldsymbol{B}$ & $\boldsymbol{C}$ & $\boldsymbol{D}$ & $\boldsymbol{E}$ & Moyenne \\
\hline Je & $21,7 \%$ & $14,7 \%$ & $21,6 \%$ & $29,8 \%$ & $16,2 \%$ & $20,8 \%$ \\
\hline $\mathrm{Tu}$ & $10 \%$ & $12,9 \%$ & $16,4 \%$ & $19,5 \%$ & $13,3 \%$ & $14,42 \%$ \\
\hline
\end{tabular}

Peut-on faire l'hypothèse que chez les enseignants du collège, il y aurait une forme de dominance $\mathrm{du}$ «je » professorale? Nous posons effectivement cette hypothèse comme susceptible de s'appliquer à la comparaison maternelle vs collège quand à l'utilisation de l'interpellation en forme de tu. En maternelle le je et le tu sont équilibrés, au collège le je professoral domine (nous avons vérifié que l'usage du je est effectivement davantage sur celui du professeur -seules 57 occurrences sur les 451 sont le fait des élèves-).

\subsubsection{Graphe typique autour des emplois du pronom interpellatif $t u$}

11 La répartition des emplois du pronom interpellatif tu apparaît différente lorsqu'on compare la maternelle et le collège. Il semble que l'enseignant en maternelle utilise l'adressage au tu de manière ponctuelle quand l'enseignant de collège l'utilise régulièrement. On peut avancer à ce stade l'hypothèse que l'adressage au tu n'a pas le même rôle fonctionnel dans l'enchainement interlocutoire dans chacun des contextes (maternelle vs collège).

\section{Graphe typique en maternelle}

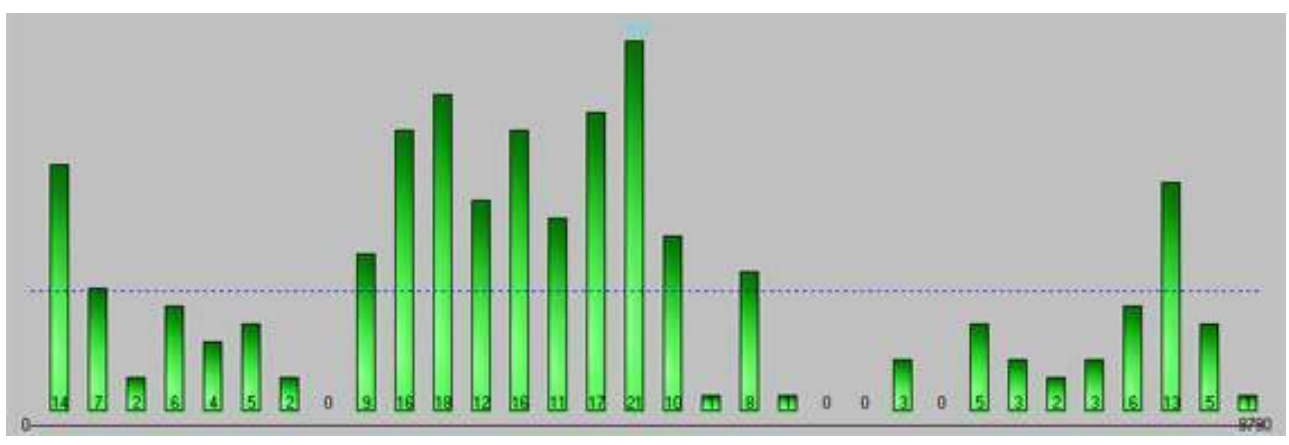

12 Commentaire : on repère dans cette répartition de l'emploi des tu le fait que 3 épisodes se distinguent tout au long de la leçon (oral réflexif).

Graphe typique au collège

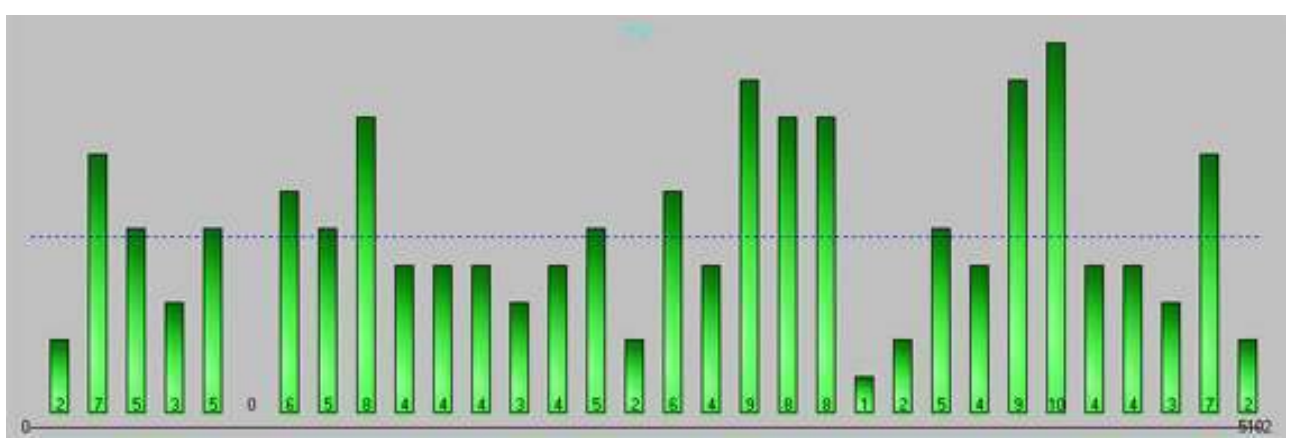

13 Commentaire : l'emploi du pronom interpellatif tu est plus régulier dans cette séance représentative des emplois au collège. L'enseignant s'adresse à l'élève tout au long de l'avancée dans son cours sans qu'on puisse distinguer des épisodes. 


\subsubsection{Marques typiques d'adressage en maternelle et au collège}

14 Lorsque l'on étudie la différence entre la maternelle et le collège, on remarque très vite que l'emploi d'un adressage à l'aide du prénom des élèves domine davantage en maternelle. D'autre part, on remarque concernant la répartition de l'emploi relatif du je comparé au tu que la répartition tout au long du cours n'est pas la même entre le collège et la maternelle.

Graphe typique d'emploi du $t u$, des prénoms et du je en maternelle

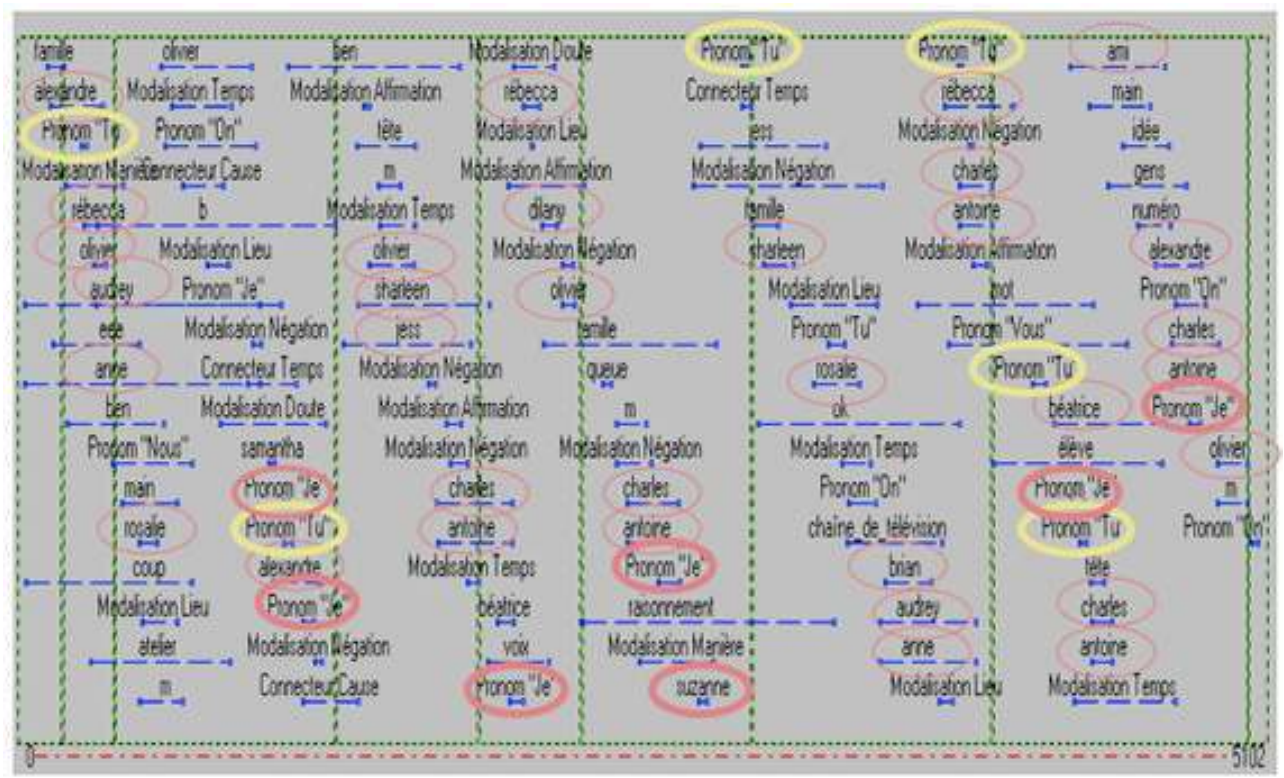

15 Légende : Le graphe se lit de droite à gauche (avancée dans la séance de cours) et de haut en bas (avancée dans un épisode thématique).

16 Commentaire: L'enseignant, dans cette séquence typique du comportement en maternelle, alterne l'adressage au tu avec l'adressage utilisant le prénom de l'élève, et il s'immisce dans la conversation scolaire plutôt en fin d'épisodes. 


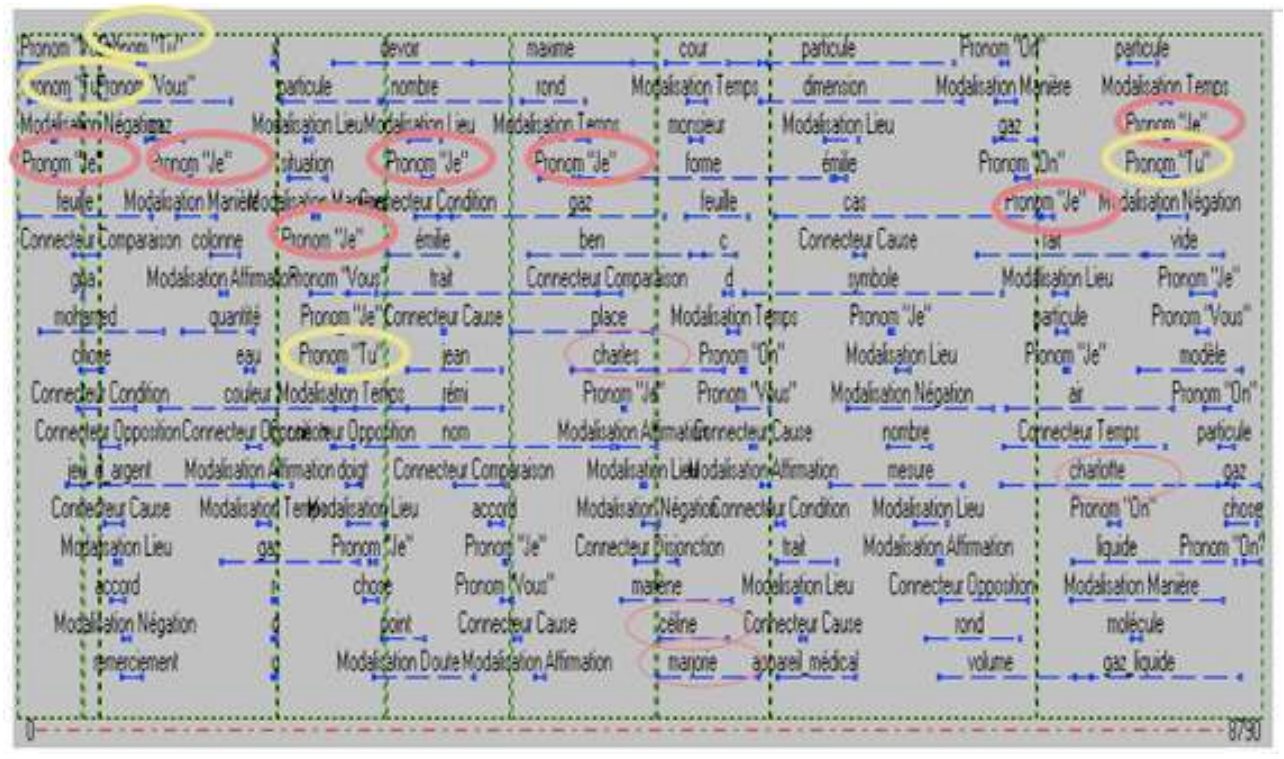

17 Légende : Le graphe se lit de droite à gauche (avancée dans la séance de cours) et de haut en bas (avancée dans un épisode thématique).

18 Commentaire: L'enseignant, dans cette séance typique du comportement en collège, utilise très peu l'adressage à l'aide du prénom des élèves, et emploie massivement le je en début de séquence.

19 Si l'on compare le comportement typique des enseignants en maternelle et au collège, on voit bien apparaitre des emplois différenciés dans les marqueurs d'adressage classique tu + Prénom et l'emploi du je marquant la présence du maître dans l'échange. En maternelle l'enseignant apparaît pour clore les épisodes thématiques; au collège il les introduit. Précisons que le logiciel Tropes délimite ces épisodes non seulement en fonction du thème mais aussi en fonction de l'avancée argumentative. Ainsi en maternelle c'est l'élève qui ouvre l'argumentation quand au collège c'est l'enseignant qui le fait.

\subsection{Sélection d'épisodes interpellatifs}

20 Sur la base de cette étude préliminaire, de laquelle se dégagent quelques hypothèses de travail autour de l'interpellation en classe, nous avons établi nos critères pour délimiter ce que nous nommons des «épisodes interpellatifs ». Un épisode est considéré à partir du relevé d'un premier indicateur (présence de tu ou du vous, du prénom ou d'un appellatif désignant l'enseignant : monsieur, madame) et est délimité à partir du traitement du thème de la conversation scolaire (question clairement adressée à un individu et représentant un thème nouveau dans le déploiement du discours). Il est difficile de délimiter correctement l'épisode. Dans chacun des exemples ( $\left.n^{\circ} 1 \mathrm{à}^{\circ} 4\right)$, nous avons mis en gras les indices que nous relevons en rapport avec l'acte de langage de l'interpellation. Certains indices, l'emploi du moi-je par exemple, seront repris dans l'étude d'orientation morphosyntaxique.

Exemple ${ }^{\circ} 1$ (épisode en maternelle) :

Ens : des mots méchants. Ok. Camille

Exemple $n^{\circ} 2$ (épisode en maternelle) 
Ens: ok, est-ce qu'il y en a d'autres qui ont des idées par rapport à ça? Oui Albert.

Albert :quand tu t'es fait mal, quand tu as bien mal à quelque part, ben t'as le droit de pleurer parce que ça fait mal, c'est sûr que t'as le droit de pleurer.

Ens: et quand tu as mal à ton cœur, est-ce que tu as le droit de pleurer?

Albert : oui parce que ça fait bien mal.

Ens : mais qu'est-ce qui est pire? D'avoir mal à son corps ou d'avoir mal à son cœur? Qu'est-ce qui est plus difficile pour vous?

Albert: moi je sais. Moi ça mon cœur.

Ens : ton cœur c'est plus difficile.Pourquoi?

Albert: parce que le cœur c'est ça qui est le plus important dans la vie. C'est ça qui fait respirer.

Ens : pourquoi c'est plus important? C'est ça qui nous fait respirer. Mais là vous parlez du cœur physique qui nous fait respirer qu'on a déjà senti ici quand on a mis note main ici pour sentir notre cœur qui respire.

21 Le premier épisode interpellatif est très court (exemple $n^{\circ} 1$ ). Pour le deuxième épisode (exemple $n^{\circ} 2$ ), tout aussi caractéristique de la maternelle, on note la longueur de l'épisode. Cette longueur provient du fait que l'enseignant poursuit le dialogue avec l'élève Albert. Ceci explique à rebours le fait que les épisodes interpellatifs sont locaux en maternelle (présence de périodes où l'adressage au tu est concentré), alors qu'ils sont réguliers au collège (périodes régulièrement distribuées; voir plus haut l'analyse automatisée avec Tropes.).

Exemple $n^{\circ} 3$ (épisode en collège)

Ens: Alors à votre avis... il faut trouver une expression

Éleve: la particule

Ens: Fanny oui?

Exemple $\mathrm{n}^{\circ} 4$ (épisode en collège)

Elève: Et comment ça a pas un nom Monsieur?

Ens: oui ça s'appelle le vide. Allezdonc vous répondez aux questions...

Légende: lorsque l'élève n'a pas été repéré, on note seulement élève. Lorsque c'est le même élève qui parle et qui a été repéré dans la transcription, on note le prénom (cf. ci-dessus en maternelle, Albert).

On remarquera la brièveté des épisodes au collège (exemple $n^{\circ} 3$ et $\left.n^{\circ} 4\right)$. Le dialogue se clôt dans l'échange binaire ou ternaire. Si on compare les épisodes relevés en maternelle et au collège, on note ainsi une grande variabilité en longueur en maternelle, et une forme d'homogénéité autour de la brièveté au collège. En revanche le nombre d'épisodes, quelle que soit la longueur, permet de comparer les corpus, au-delà de cette différence qualitative. Nous comparons donc nos deux corpus quant au nombre d'épisodes relevés (selon nos critères).

Nombre d'épisodes interpellatifs relevés en maternelle et au collège

\begin{tabular}{|c|c|}
\hline Matemelle : Séances de $40-45$ minutes & Collège : Séances de 50 minutes \\
\hline GB $: 28 / 33$ & A $: 32$ \\
\hline LO $: 24 / 34$ & B $: 66$ \\
\hline GZ $: 26 / 29$ & C $: 64$ \\
\hline SW $: 26 / 59$ & D $: 121$ \\
\hline & E $: 106$ \\
\hline
\end{tabular}

Légende : GB : 28/33 indique qu'il y a 28 épisodes dans la séance $n^{\circ} 1$ et 33 dans la séance $n$ ${ }^{\circ} 2$.

$\mathrm{Au}$ vu de ce relevé on remarque qu'il existe une très bonne homogénéité dans le corpus de maternelle : les enseignants, quelle que soit la séance, usent d'une moyenne d'épisodes avoisinant les 30 pour 40 minutes d'interaction. En revanche, au collège, sur 50 minutes 
de cours, les enseignants se différencient clairement les uns des autres. Est-ce dû à leur relative inexpérience du métier? Des études complémentaires devront confirmer les tendances que nous mettons ici à jour. Nous préciserons que le seul écart constaté dans les séances de maternelle ( $\mathrm{SW} \mathrm{n}{ }^{\circ} 2: 59$ épisodes) peut être mis en rapport avec le fait que l'enseignant a eu du mal à conduire la séquence. L'adressage à l'élève lors de cette séquence est plus capricieux, perturbé. On peut avancer l'hypothèse corrélative, pour le collège, que l'accroissement des épisodes interpellatifs serait le fait de difficultés rencontrées dans l'avancée du cours (perturbation). En ce cas les enseignants A et D seraient très différents, ce qui est vérifié par ailleurs (Auriac-Slusarczyk \& Morge, soumis). On peut aussi évoquer l'hypothèse d'un effet de genre entre les leçons du collège (cours classiques : construction d'une réponse collective consensuelle, juste et prévisible) et les oraux de type réflexifs de la maternelle (cours plus a-typique : construction d'une discussion collective respectant les avis de chacun). C'est sur la base de cette délimitation d'épisodes que les études dans la double perspective pragmatique et morphosyntaxique ont été conduites. Dans chacune des perspectives, on a cherché à faire apparaitre la spécificité des épisodes, les éventuelles régularités, comme les différences entre la maternelle et le collège lorsque c'était le cas. Ce relevé d'indicateurs nous a rendus particulièrement sensibles : aux phénomènes d'auto-interpellation (moi-je), à la présence de mouvements pro- ou rétro- actifs (brièveté versus longueur, homogénéité versus variété), à l'envergure des épisodes (organisation textuelle, récurrence de formules en c'est/ça), aux modalités de repérage plus ou moins explicite de l'individu ciblé par l'interpellation (explicite : prénom-; implicite : nouveau thème abordé), à l'équilibrage des adressages en tu ou en vous ou sous forme moins individualisée (formule du genre : les gens).

\subsection{Etude pragmatique}

25 L'analyse d'orientation pragmatique privilégie, dans la lignée du modèle d'analyse interlocutoire (Trognon, 1999, Trognon \& Batt, 2007) développé par l'école nancéenne, l'étude de l'enchaînement discursif. Dans ce cadre, nous sommes vigilants tant aux marques indicielles typiques de l'interpellation ( $\mathrm{Tu}$, prénom) qu'aux contextes d'emploi qui caractériseraient l'avènement de ces marques et qui permettent de toucher à des éléments invariants dans le procédé d'enchaînement conversationnel. Dans quel contexte l'enseignant et les élèves utilisent l'adressage à autrui? Peut-on qualifier fonctionnellement ces contextes interlocutoires?

\subsubsection{Les mouvements rétro- et pro- actifs}

26 En référence aux travaux de Roulet (Roulet \& al. 1985, Roulet, 1999), nous nommons mouvements rétroactifs les espaces interlocutoires où l'interpellation renvoie à un discours proféré avant la marque interpellative: ainsi en est-il quand l'enseignant interpelle un élève pour lui demander de préciser ce qu'il vient de dire. Nous nommons mouvement proactif l'espace interlocutoire où la marque interpellative (tu, prénom, autointerpellation je) fait référence à ce qui va se passer ensuite dans la conversation scolaire. 
Mouvements pro- et rétroactifs en maternelle

\begin{tabular}{|l|c|c|c|c|}
\hline & \multicolumn{2}{|c|}{ Proactif } & \multicolumn{2}{c|}{ Rétroactif } \\
\hline Corpus/Séance & Séance 1 & Séance 2 & Séance 1 & Śance 2 \\
\hline GZ & 12 & 11 & 31 & 38 \\
\hline LO & 14 & 22 & 39 & 35 \\
\hline SW & 15 & 60 & 30 & 82 \\
\hline GB & 22 & 37 & 25 & 85 \\
\hline TOTAL & \multicolumn{2}{|c|}{133} & & 365 \\
\hline
\end{tabular}

27 Commentaire : il y a une dominance des mouvements rétroactifs sur les mouvements proactifs.

Mouvements pro- et rétroactifs au collège

\begin{tabular}{|c|c|c|}
\hline Enseignants/Séance unique & Proactif & Rétroactif \\
\hline A & 38 & 32 \\
\hline B & 87 & 53 \\
\hline C & 51 & 73 \\
\hline D & 139 & 113 \\
\hline E & 86 & 76 \\
\hline TOTAL & 401 & 347 \\
\hline
\end{tabular}

Commentaire : il y a un équilibre entre les mouvements rétroactifs et proactifs dans les épisodes interpellatifs.

En fait, il apparaît clairement qu'entre la maternelle et le collège la progression interlocutoire n'opère pas de la même manière. Dans le cas de la maternelle, l'enseignant fait trois fois plus qu'au collège retour régulièrement sur ce qui a été dit (enchaînement rétroactif). Ce n'est pas le cas au collège, où l'enseignant utilise à parts égales la possibilité d'enchaînement sur le 'déjà dit' (rétro-) et le 'à dire' (pro). On remarque que dans le cadre de la séance $n^{\circ} 2$ du corpus SW, cas où la séance était perturbée (voir plus haut) l'enseignante use davantage d'enchaînements de type proactif puisque l'équilibre rejoint la tendance mise en évidence au collège.

\subsubsection{Les rapports de places}

L'étude systématique des rapports de places, souvent pensée comme déséquilibrés au profit de l'enseignante, est une voix d'étude classique des corpus scolaires (François \& al., 1990). Nous n'envisageons pas ici le rapport de places dans ce sens. Nous avons seulement cherché à creuser l'hypothèse d'existence d'une stratégie d'emploi pronominale particulière et différente entre le corpus maternelle et le corpus collège. Nous nous sommes centrés sur les emplois respectifs du je et du tu par l'enseignant comme par l'élève, pour mieux comprendre ou seulement mettre en évidence ce qui pointait dans l'étude automatisée. L'étude plus approfondie du fonctionnement de l'enchaînement interlocutoire au sein des épisodes dans chacun des contextes (maternelle vs collège) met en évidence des différences. L'initiative de l'adressage revient majoritairement au maître en maternelle; elle est le fait de l'élève en collège (voir ci-dessous les exemples caractéristiques du phénomène).

Exemples extraits du corpus Maternelle : emplois caractéristiques de l'adressage :

tu/je/Prénom 


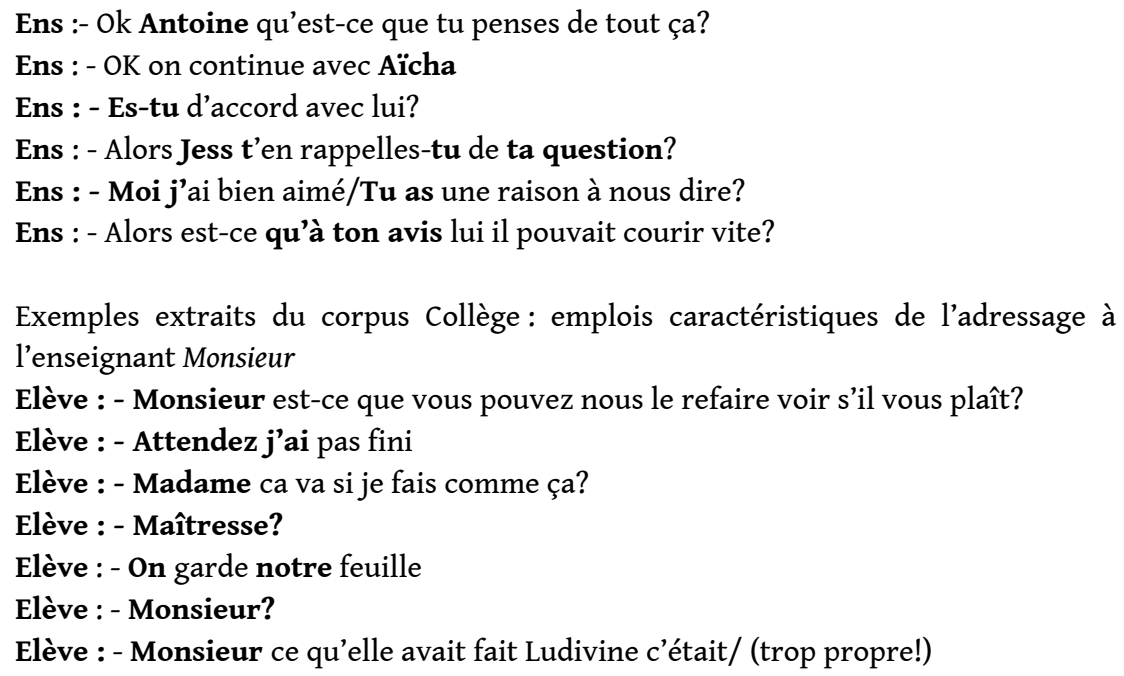

31 Ce type de renversement au profit de l'élève en collège peut surprendre. On peut aussi chercher à y déceler une logique interlocutoire. En maternelle le rapport de places va ainsi dans le sens Enseignant = > Elève. C'est l'enseignant qui sollicite régulièrement (voir l'emploi massif des Prénoms, analyse automatisée, Tropes). Cette stratégie, de la part de l'enseignant, aboutit à ce que l'élève se situe, et l'acte réactif typique de l'élève de maternelle est, pour exemple : «ben moi je peux pas dire non parce que je sais pas ». On peut dire que le je de l'élève est implicitement toujours interpellé par l'enseignant. L'élève le sait, et y répond, quand bien même justement il n'a pas la réponse, en disant : « Moi je sais pas». Au collège, c'est pratiquement l'inverse, c'est l'élève qui panique et qui interpelle alors l'enseignant: Monsieur, Madame, Maitresse. Ces interpellations sont toujours associées à une déroute chez l'élève : «j'ai pas fini ", « où met-on la feuille?", « je n'ai pas bien vu ». En somme l'élève de collège tente de ralentir le rythme du cours. Fonctionnellement, lorsque l'interpellation apparaît, le rapport de places est alors plutôt du type Elève => Enseignant. C'est comme si l'enseignant oubliait le je de l'élève. La classe est plutôt conduite au nom du 'vous', comme en témoigne l'énoncé caractéristique du professeur de collège : "Alors est-ce qu'il y a quelque chose d'autre? Réfléchissez-bien ». Il semble qu'en maternelle l'enseignant suive une logique où il interpelle l'élève au cas par cas, et tente de mettre en place un rapport à l'individu-élève ("Ens: - s'il n'a pas écouté les règlements parce que c'est pendant qu'il est en train de réfléchir ailleurs par exemple bien lui il n'est pas en train de faire son travail ç'est ça que tu dis? »:l'enseignant va chercher l'individu). Au collège, il semble que l'enseignant entre dans un rapport avec l'élève générique -élève lambda- ( $E n s:-j e$ vous ai dit où dans les enceintes où on faisait l'air il fallait une telle pression pour maintenir le liquide que l'homme ne peut pas survivre à l'intérieur donc tu peux pas respirer puisque tu peux »: l'enseignant s'adresse à l'élève comme un représentant de l'humain, au sens générique, «tu peux pas respirer » : quel que soit l'élève considéré, c'est vrai.). Le cours suit alors un parcours de type plus logique que chronologique (comparé au cas par cas de la maternelle) : l'élève doit interrompre l'enseignant et le déroulement du cours en disant: «je sais pas moi »! Cette formule est un véritable «appel au secours». De nombreux éléments apparus dans l'étude d'orientation pragmatique sont confirmés et complétés par l'étude d'orientation morphosyntaxique. 


\subsection{Etude morphosyntaxique}

32 L'on pourrait avoir des intuitions sur les phénomènes morphosyntaxiques que peuvent convoquer les situations d'interpellation. Riches de ce corpus, nous avons voulu nous garder d'une démarche peu vérifiée et montrer, à partir de faits précis, qu'il existe, pour certains points de la langue, des tendances et des particularités d'emplois (fréquence, distribution) liées à la situation d'interpellation. Par ailleurs, l'approche morphosyntaxique nous semblait bien adaptée à ce corpus; il eût été plus difficile et probablement moins pertinent de faire une description comparative maternelle/collège sur le lexique par exemple.

\subsubsection{Les schémas syntaxiques}

Comme il a été précisé précédemment, les enchaînements rétroactifs qui participent de la reformulation sont majoritaires en maternelle et bien plus longs qu'au collège. C'est pourquoi nous avons centré notre attention sur le corpus maternelle pour faire apparaître :

- que certains schémas syntaxiques interviennent très régulièrement dans la reformulation d'un discours déjà proféré pour lui donner une sorte de reconnaissance;

- que lorsque l'enseignant fait retour sur ce qui a été dit, il utilise des outils morphosyntaxiques qui contribuent à la construction du texte et par conséquent à la construction et à l'organisation de l'épisode rétroactif. trouve assez systématiquement des «reprises» en c'est ou ça de type: (a) dire tu es méchant c'est être méchant ; (b) poser une question ça veut dire qu'on parle à quelqu'un ; (c) moi j'aimerais savoir pourquoi ces gestes-là puisces mots-là c'est méchant. Il s'agit alors de dislocations de construction verbales à l'infinitif (a), (b) ou de syntagmes nominaux (c) qui ont été déjà mentionnés par l'élève. Il est intéressant de voir que c'est un procédé systématique auquel on peut attribuer une valeur pragmatique : la reprise en c'est/ça donne un caractère définitoire après-coup à la construction (Blanche-Benveniste, 1997) et l'enseignant valide -par cet opérateur pragmatique- les thèmes associés jusque-là à l'enfant. Mais plus que cela, la dislocation est un «outil » qui permet un traitement métalinguistique sur le dire. Ainsi dans l'exemple (c), le sens ne serait pas le même avec une construction directe de type « ces mots-là sont méchants ». La reprise en c'est induit qu'il s'agit de "dire ces mots-là ». La dislocation insiste sur le dire; ce ne sont pas les mots directement qui sont visés.

\section{Progression du texte}

Les passages où l'enseignant reformule rétroactivement les propos de l'élève sont remarquablement construits et organisés, et là aussi on peut considérer certains faits morphosyntaxiques comme des outils qui contribuent à la progression et à l'organisation de ces espaces interlocutoires.

Exemple :

Ens : - quand tu dis ça qu'est-ce que tu es en train de dire tu es en train de dire que c'est pas pareil pour les adultes et pour les enfants c'est-à-dire que les adultes font des choses et ils disent aux enfants de faire des choses puis eux ils ne le font pas ils 
disent aux enfants qu'ils n'ont pas le droit de courir puis eux ils courent c'est ça que tu veux dire du point de vue lexical. Pourtant il suffit de le présenter selon une analyse en grille (C. Blanche-Benveniste et alii 1990), c'est-à-dire d'en donner une représentation à la fois syntagmatique et paradigmatique pour voir apparaitre les structures grammaticales majeures qui organisent cette production orale et participent à la construction du discours.

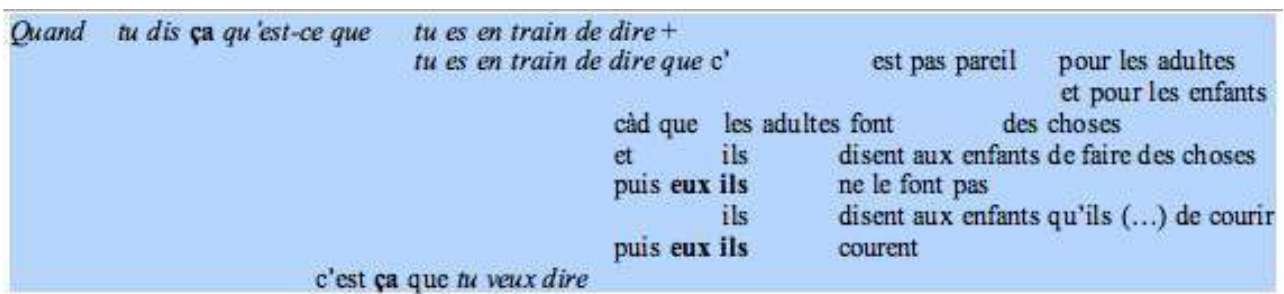

41 L'enseignant questionne dans un premier temps l'élève sur le contenu exact de son propos avec des répétitions (cf. Tannen, D., 1987) : quand tu dis ça qu'est-ce que tu es en train de dire. Àce moment là, le ça est assez globalisant; il réfère aux propos de l'élève dans un énoncé donné. Puis l'enseignant reprend mais précise en même temps le contenu de ce dire en développant l'axe syntagmatique : tu es en train de dire que c'est pas pareil pour les adultes et pour les enfants. Puis, c'est-à-dire que introduit un développement paradigmatique qui repose sur des répétitions lexicales ou syntaxiques font des choses, disent aux enfants de faire des choses et des contrastes de modalité font/ne font pas; ils n'ont pas le droit de courir / ils courent. Notons par ailleurs que le centre du propos « la différence entre les adultes et les enfants » se retrouve aussi dans la dénomination : pour marquer l'opposition entre les deux, la dislocation du sujet eux-ils est plutôt pertinente puisque jusque-là, les sujets ne sont pas disloqués : les adultes font, ils disent. Enfin pour clore ce passage l'enseignant en arrive à c'est ça que tu veux dire. Une construction verbale qui rompt avec la précédente tu es en train de dire que $e^{3}$. Le "ça » boucle l'épisode mais il est alors sémantiquement chargé de toute la démonstration.

42 Ainsi, l'organisation globale du texte est centrée sur une opposition adulte / enfant. Àpartir de là le texte joue entre autres sur des contrastes ils/eux-ils etc. Mais tous ces critères syntaxiques prennent place dans le schéma d'organisation du discours plus général et fonctionnent comme des outils morphosyntaxiques qui s'inscrivent dans cette opposition entre les adultes et les enfants.

\subsubsection{La Distribution des pronoms : Je et Moi Je}

43 Nous ne pouvions aborder l'interpellation sans parler des pronoms de 1 ère personne. En revanche, ce que nous ne pouvions pas prévoir, c'était les paramètres qui détermineraient la distribution de je et de moi je. Il nous est apparu dans les deux corpus que la répartition entre je et moi je est conditionnée par différents critères. Tout d'abord, selon que le discours est monologal ou dialogal. Ensuite, quand il est dialogal, on observe des différences selon que c'est une initiative de prise de parole (mouvement proactif) ou une réponse à une sollicitation (mouvement rétroactif). Mais il est apparu aussi un critère inattendu : le lexique verbal. En effet, il se passe quelque chose de remarquable avec le verbe savoir; verbe plutôt présent dans ces échanges élève/enseignant puisque c'est le 2 ème verbe à apparaître avec moi je dans les corpus retenus. 

une comparaison entre les deux corpus (maternelle-collège). Ces tendances témoignent d'une utilisation moins libre que ce que l'on pourrait croire de la morphosyntaxe. Elles pourraient être mises en relation avec le genre de discours décrit, d'autant que les faits ne corroborent pas les tendances observées pour le français parlé tout venant. Ainsi, en maternelle, employé avec moi-je le verbe savoir est très régulièrement sous une modalité positive, alors qu'employé avec je, il est sous une modalité négative ${ }^{4}\left(\right.$ exemple $^{\circ} 3$ et $n^{\circ} 4$ )

Exemple $n^{\circ} 3$ (maternelle) :

Ens : - Qu'est-ce que c'est, son idée? Vous vous souvenez de l'idée de Laurie? (signe de tête négatif de Camille)

Mathew: - Moi oui

Ense : - C'est quoi l'idée de Laurie?

Mathew: - Elle dit que t'arrêtes

Ense :- Puis Laurie, toi tu dirais ça, pourquoi tu dirais cela... à son amie.

Laurie : - Je ne sais pas

Ense : - C'est ça que tu penses qui est le mieux?

Exemple $\mathrm{n}^{\circ} 4$ (maternelle)

Maxime :- bah, je sais pas.

Ens :- qu'est-ce qui se passe quand tu es avec elle?

Aïcha :- moi je sais pourquoi. Parce qu'elle nous le dit!

Maxime : - bah, je sais pas.

Aïcha : - moi je sais! Parce que normalement quand ils s'aiment, on le dit vite. 
affirmative/négative. Il est intéressant de noter (cf. exemple $\mathrm{n}^{\circ} 1$ ) que l'élève ne « redouble» le sujet que pour parler de lui. On peut avancer qu'il a bien besoin de se distinguer des autres personnages : un ami, ma mère dans un contexte de comparaison : elle lui donnerait/j'en aurais pas. Mais il est vrai qu'en phase narrative, dès qu'il n'y a pas de contraste, d'indice de comparaison, de jeu de modalité, alors le moi je n'est plus convoqué (exemple $n^{\circ} 2$ ).

Exemple $\mathrm{n}^{\circ} 1$ (maternelle) :

Elève: c'est comme si ma mère c'est comme si ma si un ami veut venir chez moi puis

elle lui donnerait du gâteau puis moi j'en aurais pas ça serait injuste

Exemple $\mathrm{n}^{\circ} 2$ (maternelle) :

Mathew (à la classe) : À un moment donné j'avais invité un de mes amis puis, un moment donné là, là, c'est une fille. Elle allait à ma garderie avant. J'avais fait de quoi de pas de gentil. Maman, elle m'a envoyé puis elle, elle a pleuré jusque... j’y ai fait une surprise. Je lui ai trouvé un cadeau dans, dans...

Voix de petite fille: ... dans ta chambre...

Mathew: ... dans ma chambre. Je lui ai donné un toutou.

L'intérêt que nous avons porté à l'utilisation du pronom de $1^{\text {ère }}$ personne sous la forme je ou moi je dans les épisodes interpellatifs est double: nous avons vérifié a) que des paramètres complexes interviennent dans la forme de réalisation du sujet; et b) que ces espaces interlocutoires précisent ce que nous savons de la distribution de ce sujet (BlascoDulbecco 2004, 2006) en français tout venant.

\subsubsection{Formes « lexicales » d'adressage collectif}

51 Les tendances observées dans l'utilisation de formes lexicales complètent l'interprétation pragmatique. On pourrait proposer de dire que l'adressage au groupe se fait avec des termes davantage individualisants en maternelle (quand l'enseignant s'adresse au groupe) et davantage globalisants en collège, et ce, en fonction de l'espace interlocutoire dans lequel intervient l'interpellation. En effet, pour interpeller la classe, l'enseignante de maternelle emploie il y en a les autres. Elle emploietrès minoritairement, et semble-t-il seulement dans les épisodes rétroactifs, tout le monde. La classe n'est donc pas envisagée dans sa totalité mais par groupes, dès lors que l'enseignant s'adresse ou sollicite les élèves.

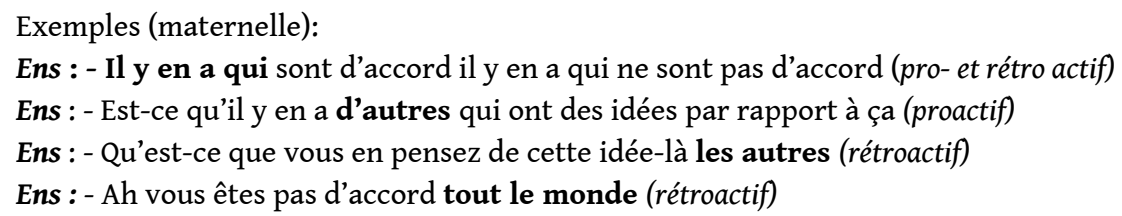

En revanche, au collège, l'interpellation de la classe se fait très majoritairement avec quelqu'un quand l'enseignant sollicite les élèves en les invitant à participer. C'est un pronom très indéfini puisqu'il renvoie à un individu anonyme (série d'exemples $n^{\circ} 1$ ) par opposition à un groupe défini de personnes qui auraient déjà parlé (cf. maternelle plus haut). Lorsqu'il ne s'agit plus d'une sollicitation mais d'un vif souhait qui ne se prête pas à l'individualisation et que la séquence doit avancer, alors l'adressage est réalisé par tout le monde (série d'exemples $\mathrm{n}^{\circ} 2$ ).

Exemples $n^{\circ} 1$ (collège):

Ens : - quelqu'un a autre chose à ajouter

Ens : - quelqu'un veut aller au tableau 
Exemples $n^{\circ} 2$ (collège):

Ens : - on notera tout à la fin bon maintenant pour ce qui n'a pas changé la quantité de gaz tout le monde est d'accord?

Ens : - une fois que la partie au propre est notée vous regardez l'activité 2 tout le monde y est ? tout le monde comprend la consigne? monde a quelque chose à ajouter? ${ }^{*}$ tout le monde passe au tableau? Mais dès lors que l'enseignant se place dans le groupe, et ne le sollicite plus pour désigner un groupe particulier (sous groupe classe) alors il utilise des lexèmes globalisants: les gens, les personnes (exemple $\mathrm{n}^{\circ} 3$ ).

Exemple $n^{\circ} 3$ (collège) :

Ens : - attendez on laisse il y en a que trois il y a trois personnes qui ont été désignées donc il n'y a plus personne qui passe on laisse faire au tableau ce que les gens ont à faire et ensuite on discute quand ils ont fini

On peut faire l'hypothèse qu'en maternelle, l'enseignante s'adresse à " chaque élève » même s'il peut s'agir de sous groupes- sauf quand il y a consensus dans la classe : «vous êtes pas d'accord tout le monde ». En collège, l'enseignant ne fait pas cette distinction, l'élève sollicité peut être individualisé ou ramené au groupe et cela semble motivé par la réponse que l'enseignant attend, ce peut être quelqu'un ou tout le monde. Mais dès qu'il s'agit de nommer une partie de la classe aux autres élèves, alors l'enseignant passe par des lexèmes très désindividualisants comme les gens, personne.

\section{Synthèse des résultats et conclusion}

Pour synthétiser, nous présentons dans le tableau suivant les principales tendances que la comparaison maternelle vs collège nous a permis d'établir.

Comparatif Maternelle vs Collège sur l'ensemble de nos indicateurs

\begin{tabular}{|l|l|}
\hline \multicolumn{1}{|c|}{ Maternelle } & \multicolumn{1}{c|}{ Collège } \\
\hline 1. Homogénéité entre enseignants & 10. Variabilité entre enseignants \\
2. Emploi de $T u=J e$ & 11. Emploi de $J e>T u$ \\
3. Interpellation au tu locale située, & 12. Interpellation au tu régulière \\
individualisée & 13. Tu générique \\
4. Prénom sur-employé & 14. Épisodes brefs \\
5. Épisodes variés & 15. Mouvements proactifs-rétroactifs \\
6. Mouvements rétroactifs & 16. Adressage collectif anonyme: tout le \\
7. Reprises en c'est/ça & monde, les gens \\
8. Moi-je/ je & \\
9. Ilyen a, les autres & \\
\hline &
\end{tabular}

Pour conclure, nous dirons que l'interpellation pourrait bien permettre de pister une forme de stratégie discursive centrale utilisée par l'enseignant pour faire classe, c'est-àdire pour faire progresser l'interaction scolaire. En ce sens, décrire les modalités interpellatives d'un enseignant dévoile, en partie, sa manière de faire la classe. Dans le cadre restreint de notre corpus, nous avançons qu'en maternelle, l'enseignant s'immisce dans le monde intellectuel de l'élève: grâce à des reformulations provoquées par l'enseignant (rétroaction dominante) qui propulsent la progression du discours scolaire, le texte prend peu à peu une structure complexe et très organisée. En maternelle l'enseignant travaille les questions et le thème proposé par les élèves (rétroactions, reprises en c'est/ça) est repris au sein d'un "véritable " dialogue. Au collège, par comparaison, l'enseignant semble faire comme un «pari » (l'équilibre des types proactif 
et rétroactif au sein des enchaînements interlocutoires est assuré) sur ce que l'élève (au sens générique) ou le groupe (désigné par plusieurs formules de type désindividualisant : des gens) peut lui apporter, pour avancer dans le cours. Dit autrement, en contexte maternelle, l'enseignant pratique davantage le jeu stratégique de l'intrusion (l'enseignant domine tout en tenant compte de l'élève), et la discussion se structure autour d'un cheminement discursif où le $\mathrm{Je}$ du professeur enquête sur le $\mathrm{Tu}$ de l'élève. Dans le contexte du collège, l'enseignant pratique le jeu de l'épreuve (pour les deux parties: enseignant et élève), où ce serait l'énigme (du point de vue de l'élève) qui domine, ce qui se traduit par l'organisation d'un cheminement discursif où c'est le $T u$ de l'élève qui devine ce que le Je du professeur attend. Les professeurs se différencient dans la gestion de cette épreuve de type devinette.

Àl'issue de cette étude comparant les manières d'interpeller en classes de maternelle et de collège, nous restons prudents sur nos interprétations. Une généralisation de nos résultats nous paraît prématurée et nous pensons qu'il conviendra de vérifier s'il n'existe pas derrière ces contrastes repérés des effets dus au genre de conversation potentiellement différent entre le corpus maternel (oraux réflexifs) et le corpus collège (leçon ordinaire). Nous proposons toutefois, dans le cadre restreint de notre étude, d'associer chacun des niveaux étudiés (maternelle et collège) aux synonymes repérés comme faisant partie du champ lexical de l'interpellation.

Synonymes d'Interpeller : A postrophe (1), interrogation (2), intervention (3), question (4)

(1): appel, interpellation, invective (Spécificité : Maternelle \& Collège).

(2): colle, demande, épreuve, interpellation, interrogatoire, question, questionnaire (Spécificité : Collège)

(3): action, concours, entremise, immixtion, initiative, interposition, intrusion, operation, therapeutique, traitement (Spécificité : Maternelle)

(4): affaire, charade, colle, controverse, demande, devinette, difficulté, discussion, énigme, interpellation, interrogation, point, problème, thème, torture. (Spécificité : Maternelle \& Collège).

Il semble que l'on puisse associer la maternelle et le collège (sans distinction) à l'usage de l'interpellation dans le double sens classique d'apostrophe (1) et de question (4) et par conséquent à l'ensemble des termes associés respectivement à ces synonymes (1 et 4$)$. Quelle que soit la classe, lorsque l'enseignant apostrophe (1) il questionne (4). En revanche le niveau collège serait plus représentatif d'un usage de l'interpellation lorsqu'elle prend la forme de l'interrogation (2), alors que le niveau de la maternelle serait plus représentatif de la forme d'interpellation lorsqu'elle se fait intervention (2). Des études complémentaires devront infirmer ou confirmer ces tendances. Au-delà, l'interpellation nous est apparue comme une voie d'entrée très fructueuse pour caractériser ce qui est typique des interactions didactiques. L'interpellation est une figure centrale en classe. D'ailleurs, certaines études révèlent que les élèves de collège et de lycée sont très sensibles aux modalités d'interpellation $(t u+$ nom, $t u$ + Prénom, vous + Nom, etc.) (Edouard, 2005, 104). Notre étude de corpus confirme que les modalités d'interpellation en classe ne sont pas neutres et que les recherches doivent se poursuivre en ce sens. 


\section{BIBLIOGRAPHIE}

Auriac-Slusarczyk, E., (à paraître). Les interactions scolaires : actualités et méthodes, Revue

Psychologie de l'Interaction, $\mathrm{n}^{\circ}$ spécial.

Auriac-Slusarczyk, E., (2009). Etude psychosociale d'une séquence d'enseignement de la lecture au cours préparatoire. TFE : Travail \& Formation en Education. Revue européenne interdisciplinaire de recherche.

Auriac, E., (2008). Vers une topique des discours scolaires Etude des configurations pronominales autour de quatre pronoms typiques : 'on', je', 'tu', 'il'. In Auriac, E., Baquès, M-C., Cizeron, M., Gal, N., Goigoux, R., Ria, L., Contribution du Laboratoire PAEDI à l'étude des gestes professionnels des enseignants expérimentés et débutants. Journée d'étude : La notion de gestes professionnels : entre analyse de l'activité, épistémologie et ingénierie de formation. Montpellier : I.U.F.M. de Montpellier 2, 19-21 juin 2008.

Auriac, E., (2007). Quels indicateurs retenir pour progresser dans l'étude des discours scolaires? In A. Sepcogna (Dir.), Enseigner dans l'interaction (pp. 33-56). Paris : L'Harmattan.

Auriac, E. (2007). Approche psychosociale de la discussion à visée philosophique comme praxis scolaire (élève), champ de formation (enseignant) et objet de recherche (pragmatique du discours). In M. Tozzi (Dir.), Apprendre à philosopher par la discussion : Pourquoi ? Comment? (pp. 109-122). Bruxelles : Les Presses de l'université De Boeck.

Auriac-Peyronnet, E., (2003). Comment étudier ce qu'ils disent ? Perspective psycholinguistique. In E. Auriac-Peyronnet, (Dir.). Je parle... Tu parles... nous apprenons. Figures croisées de la Coopération et de l'Argumentation. (pp. 67-84). Bruxelles : Paris : De Boeck Université.

Auriac-Slusarczyk, E. \& Morge, L. (soumis). La cohérence discursive des professeurs comme indice d'efficacité d'enseignement : étude exploratoire. Education et Didactique.

Blanche-Benveniste, Cl. \& alii (1990). Le français parlé, études grammaticales. Paris : édition du CNRS.

Blanche-Benveniste, Cl \& alii (1997). Approches de la langue parlée en français. Paris. Ophrys.

Blasco-Dulbecco, M., (2006). La description syntaxique du français à la lumière des usages oraux. Actes du Colloque International Français langue internationale, Recherche et formation, L'Europe des Langues et des Cultures. Cracovie : Université pédagogique de Cracovie 23-25 juin 2005. Synergie, 2, 19-28.

Blasco-Dulbecco, M., (2006). Propositions pour le classement typologique de quelques détachements. Information grammaticale, 109, 27-33.

Blasco-Dulbecco, M., (2004). Quelques éclairages sur le sujet de type moi je à l'oral. Recherches sur le français parlé, 18, 127-144.

Edouard, M., (2005). Premières classes. Bien débuter le métier d'enseignant en collège et en lycée. Amiens : Editions du Sceren : CRDP d'Amiens.

François, F., Bautier-Castaing., E., de Heredia-Deprez, C., Langumier, M., Nonnon, E., Van der Straten, A. \& Vasseur, M-T., (1990). Communication inégale. Heurs et malheurs de l'interaction verbale. Neuchâtel, Paris : Delachaux \& Niestlé, 
Roulet, E. \& alii. (1985). L'articulation du discours en français contemporain. Berne Francfort s/Main : Peter Lang.

Roulet, E., (1999). La description de l'organisation du discours. Saint-Jean-de-Braye : Didier.

Tannen, D., (1987). Repetition in Conversation : Toward a Poetics of Talk, Language, 63, 574-605.

Trognon, A. \& Batt, M., (2007). Comment conduire l'examen d'un fragment d'interlocution au moyen de la logique interlocutoire? In A. Specogna (Dir.), Enseigner dans l'interaction (pp. 13-33). Paris : L'Harmattan.

Trognon, A., (1999), Eléments d'analyse interlocutoire, Ch2., in M. Gilly, J-P. Roux \& A. Trognon. Apprendre dans l'interaction, coll. Langage- Cognition- Interaction, (pp. 69-94). Nancy : Presses universitaires \& Marseille : Presses universitaire de Provence.

\section{NOTES}

1. Ce corpus a été collecté grâce aux subventions du C.R.S.H., organisme de financement canadien, dans le cadre d'un projet conduit par Mme Daniel, professeur à l'Université de Montréal.

2. Ce corpus a été constitué par L. Morge dans le cadre d'une étude effectuée dans le laboratoire P.A.E.D.I. IUFM d'Auvergne. Le corpus est consultable en ligne à http://auvergne.iufm.fr

3. On passe à une autre structure grammaticale, d'où l'encadrement.

4. Dans un travail antérieur (Blasco-Dulbecco, 2004) nous avions noté un emploi remarquable de formes je sais pas moi en français parlé.

\section{RÉSUMÉS}

Les espaces interlocutoires qui correspondent à des «interpellations directes » sont étudiés comparativement en maternelle et au collège. Une étude préliminaire automatisée des corpus identifie le rôle de premiers indicateurs -pronoms je et tu et prénoms-. Une deuxième étude, pragmatique, isole les épisodes interpellatifs en extrayant les circonstances interlocutoires précisant l'emploi de ces pronoms et prénoms. Sont différenciés de cette manière les deux contextes (collège et maternelle) : 1) forte majorité d'épisodes rétroactifs en maternelle et 2) divergence fonctionnelle quant au rapport de places élèves-professeur. Une troisième étude morphosyntaxique révèle des tendances et des particularités d'emplois remarquables en langue ( c'est, moi je), l'usage de tournures caractéristiques, (les gens), des organisations textuelles complexes. Au final, on avance que la discussion scolaire construit un cheminement interpellatif où 1) en maternelle c'est le professeur qui « enquête " sur l'élève, tandis que 2) au collège c'est l'élève qui doit « deviner » ce que le professeur attend.

Interlocutory spaces corresponding to 'direct interpellation' were studied comparatively in infant and middle schools. Preliminary processing of corpora identified the role of the prime indicators - pronouns I and you and first names. A second, pragmatic study isolated interpellative episodes by extracting the interlocutory circumstances governing the use of these pronouns and first names. In this way, the two contexts were differentiated (infant and middle 
schools): (i) a large majority of retroactive episodes in infant school and (ii) functional divergence in pupil-teacher relative positioning. A third, morphosyntactic study showed noteworthy trends and specific features in language use, e.g., it's..., I-myself..., the use of characteristic expressions, e.g., people..., and complex textual organisation. We conclude that classroom discussion constructs an interpellative route where (i) in infant school the teacher 'queries' the pupil, whereas (ii) in middle school the pupil has to 'guess' what the teacher is expecting.

INDEX

Mots-clés : interpellation, corpus, pragmatique, morphosyntaxe, didactique

Keywords : interpellation, corpus, pragmatics, morphosyntax, didactics

\section{AUTEURS}

\section{EMMANUĖLE AURIAC}

Laboratoire P.A.E.D.I., IUFM d'Auvergne JE 2432. Laboratoire de psychologie Cognitive et Clinique (EA 4165, Université de Nancy 2)

\section{MYLÈNE BLASCO-DULBECCO}

Laboratoire L.R.L. (Clermont 2) et DELIC (Université de Provence) 